ArtefaCToS. Revista de estudios de la ciencia y la tecnología

eISSN: $1989-3612$

Vol. 8, No. 2 (2019), 2. ${ }^{\text {a }}$ Época, 27-52

DOI: http://dx.doi.org/10.14201/art2019822752

\title{
De la telaraña a la Web: artefactos cognitivos en animales no-humanos
}

\section{From spider web to Web. Cognitive artifacts in non-human animals}

\author{
Joan Sebastián MEJÍA RENDÓN*; Andrés CRELIER ${ }^{* *}$ \\ *Universidad de Antioquia, Colombia \\ jsebastian.mejia@udea.edu.co \\ ${ }^{* *}$ Universidad Nacional de Mar del Plata, Argentina \\ andrescrelier@gmail.com
}

Recibido: 27/10/2018. Revisado: 20/06/2019. Aceptado: 02/08/2019

\section{Resumen}

En este trabajo examinamos la noción de "artefacto cognitivo" y su posible aplicación a la técnica animal. Aunque poco se ha dicho acerca de la técnica de los animales no-humanos, los estudios de caso centrados en la capacidad de uso y fabricación de herramientas sugieren que algunos animales, además de desarrollar complejas destrezas físicas, también despliegan habilidades cognitivas. ¿Podrían las herramientas utilizadas por los animales considerarse genuinos artefactos cognitivos? En este trabajo propondremos una definición de "artefacto cognitivo" con base en la noción de "función propia", lo cual nos permitirá desarrollar una escala gradual en donde ubicaremos diversos tipos de artefactos según la preponderancia de la función cognitiva en oposición a la función práctica. Aplicando esta escala al ámbito no-humano, defenderemos la idea de que existe un ámbito técnico no-humano de fenómenos de uso y fabricación de herramientas y artefactos en el cual algunos de estos artefactos poseen una incipiente función propia de naturaleza cognitiva. Para ello, ilustraremos con ejemplos de las telas de araña, nidos y panales.

Palabras clave: Artefactos cognitivos; Animales no-humanos; Función propia; Función sistémica; Telarañas; Panales de abeja. 


\begin{abstract}
We examine the notion of cognitive artifact and its possible application to the animal technic. Although the issue of animal technic is not very much discussed, the case studies focused on the capacity of using and manufacturing tools suggest that some animals deploy cognitive skills. Could the tools employed by animals be considered genuine cases of cognitive artifacts? In this paper we propose a definition of "cognitive artifact" based on the notion of "proper function," which allows us to develop a gradual scale where we place different kinds of artifacts according to the prevalence of the cognitive function (in opposition to the practical one). Applying this scale to the non-human sphere, we defend the idea that there exists a range of non-human technical phenomena of use and artifacts and tools manufacturing, and that some of this artifacts have an incipient proper cognitive function. In order to defend these theses, we examine the examples of the cobwebs, the nests and the honeycombs.
\end{abstract}

Keywords: Cognitive artifacts; non-human animals; Proper function; System function; Cobwebs; Honeycomb.

\title{
1. Introducción
}

Gran parte de los debates recientes sobre los sistemas cognitivos se desarrolla en el marco de la denominada cognición situada (CS) surgida a mediados de los años '80, según la cual el proceso cognitivo no se realiza exclusivamente dentro de las bóvedas craneanas, sino también, en el mundo exterior (Clartcey 2009). La CS plantea que en los procesos cognitivos se incorpora el cuerpo (the embodied cognition thesis); los ambientes naturales, sociales y artificiales (the embedded cognition thesis); y adicionalmente, estos procesos se extienden y distribuyen a través de agentes y de artefactos (the extended and distributed cognition theses) (Robbins and Aydede 2009).

Como se advierte especialmente en esta última variante, la CS se constituye como un excelente marco teórico entender el rol de los artefactos en determinadas tareas cognitivas. Artefactos como los videojuegos (Tetris), los mapas, los giroscopios, los radares, los cuadernos, los diagramas, procesadores de palabras, los cuadernos de bocetos, las calculadoras y las interfaces de las computadoras han sido denominados por algunos teóricos como artefactos cognitivos (Clark 2006b; Clark and Chalmers 1998; Heersmink 2013, 2013; Kirsh 1995; Lomas 2007; Maglio and Kirsh 1992; Norman 1992; Norman, Donald, and Carroll 1991; Sterelny 2004).

Esta lista sólo incluye artefactos diseñados y usados por humanos para cumplir tareas cognitivas generalmente sofisticadas. Pero, si bien se ha discutido sobre cómo entender esta clase de artefactos, y se ha asumido que se trata de entidades 
pertenecientes a la cultura humana, poco se ha intentado reflexionar acerca de si la fabricación y el uso de artefactos por parte de diversos animales no-humanos (de ahora en adelante, animales) cumple también funciones cognitivas. Este sesgo antropocéntrico ha sido nuestra motivación principal para extender la discusión fuera del ámbito humano: se reconoce que muchas especies animales son hábiles en cuanto a la fabricación y al uso de artefactos, ¿pero, acaso estas destrezas físicas podrían involucrar elementos cognitivos?; a su vez, ¿podrían las herramientas utilizadas en ellas considerarse genuinos artefactos cognitivos?

Desarrollaremos nuestro enfoque en dos secciones principales. En la primera de ellas, propondremos una definición de "artefacto cognitivo" con base a la noción de "función propia", lo cual nos permitirá desarrollar una escala gradual en donde ubicaremos diversos tipos de artefactos según la preponderancia de la función cognitiva en oposición a la función práctica. En la segunda de ellas aplicaremos nuestras herramientas conceptuales al ámbito no-humano. Para ello, defenderemos la idea de que existe un ámbito técnico no-humano de fenómenos de uso y fabricación de herramientas y artefactos; y nos acercaremos a la tesis central de este trabajo: consistente en señalar que algunos de estos artefactos poseen una incipiente función propia de naturaleza cognitiva, algo que ilustraremos con los ejemplos de las telas de arańa, nidos y panales.

\section{Artefactos cognitivos}

\subsection{Definición de artefacto cognitivo}

Autores como Edward Hutchins han considerado a los artefactos cognitivos como objetos físicos diseñados por humanos y que tienen el objetivo de ayudar, realzar e, incluso, mejorar nuestra cognición (Hutchins, 1999, p. 126). Tareas cognitivas como navegar, calcular, medir, recordar, planear, clasificar o comparar serían en ocasiones muy difíciles de realizar si no se recurriera a los artefactos cognitivos. Una moderna computadora, una lista de compras del supermercado, un calendario o, incluso, un hilo atado en un dedo para recordar información son sólo algunos ejemplos de estos artefactos. Teniendo en cuenta esto, los intentos de definición que se encuentran en la literatura concuerdan en que la principal característica de los artefactos cognitivos es su capacidad de procesar información (Brey 2005; Donald 2010; Heersmink 2018; Kirsh 1995; Nancy and Wendy 2003; Sterelny 2003).

Donald Norman $(1991,1992)$ propone una definición más específica al afirmar que los artefactos cognitivos son dispositivos artificiales diseñados para mantener, mostrar u operar sobre determinado tipo de información en orden a cumplir con una función representacional que mejora nuestra cognición. Esta función representacional hace referencia a la capacidad que tienen estos dispositivos para referirse al mundo (aboutness). Para este autor, los artefactos cognitivos pueden poseer un nivel representacional externo o superficial, como la desplega- 
da en un libro abierto o un mapa, y otro interno, como la información contenida dentro de un dispositivo y que sólo se accede a ella mediante ciertas interfaces, así cuando debemos encender un laptop y examinar los archivos en su sistema operativo.

Pero no todos los autores concuerdan en que los artefactos cognitivos se caracterizan exclusivamente por su función representacional ligada con una estructura de este tipo. Richard Heersmink propone una clasificación más amplia que incluye artefactos cognitivos representacionales y ecológicos (no-representacionales) (Heersmink 2012). Por un lado, los artefactos representacionales como los mapas, calendarios, termómetros, tablas de colores, diagramas, modelos científicos, etc., se caracterizan porque ofrecen una representación del mundo para un agente determinado (Heersmink 2016). Por otro lado, los artefactos cognitivos ecológicos o no-representacionales se caracterizan por ayudar a realizar tareas cognitivas sin representar o referirse a un objeto determinado necesariamente. Por ejemplo, dejar constantemente las llaves del auto en un lugar para que no sea necesario recordar dónde se han dejado u organizar el espacio de trabajo de manera que la ubicación de los objetos facilite la tarea que uno está haciendo (Kirsh 1995). De esta forma, los artefactos cognitivos ecológicos codifican la información importante que luego es usada por el agente en sus tareas cognitivas. Un artefacto cognitivo ecológico puede no haber sido diseńado específicamente para una función cognitiva, pero es el agente quién intencionalmente ha codificado cierto tipo de información en el artefacto, a través de su referencia espacial y su locación (Heersmink 2013).

Asimismo, autores como James Holland, Hutchins y David Kirsh afirman que los artefactos cognitivos ecológicos también toman como referencia espacios no-físicos, sino virtuales (Hollan, Hutchins, and Kirsh 2000). Por ejemplo, cuando usamos marcadores de navegación para archivar páginas favoritas o cuando dejamos alguna página web abierta para priorizar su importancia, entonces estamos haciendo que su localización posterior sea más fácil de recordar. De esta manera, la definición de artefacto cognitivo se amplía para incluir la ubicación y referencia de elementos en espacios virtuales. Esta visión ampliada permite hablar de otros artefactos cognitivos, como las tácticas mnemotécnicas o el lenguaje (Clark 2006a), por su capacidad de ayudar a ciertas tareas cognitivas.

Lo anteriormente expuesto nos propone una visión ampliada de los artefactos cognitivos que no posee un sesgo inicialmente antropocéntrico. Según ella, los artefactos cognitivos son una clase de artefactos utilizados por agentes que los han diseńado, fabricado o adaptado (como en el caso de los artefactos cognitivos ecológicos) con el objetivo de realizar o llevar a cabo diversas tareas cognitivas (navegar, calcular, medir, recordar, planificar, diseñar, etc.). Para poder cumplir estas tareas, los artefactos cognitivos poseen una estructura representacional o ecológica que les permite procesar información proveniente del entorno. 
Sin embargo, esta propuesta de definición se enfrenta a la siguiente objeción. Puede alegarse que cualquier artefacto puede en principio utilizarse para procesar información, como el caso del hilo atado en un dedo para recordar algo determinado, o incluso que todo artefacto ya "procesa" información, como un martillo que registra la temperatura del ambiente. Esta objeción parece trivializar la idea de artefacto cognitivo desdibujando la idea de que estamos frente a una clase particular de artefactos. Para que esto último no suceda, en lo que sigue defenderemos la distinción entre función cognitiva propia, que sirve para caracterizar a esta clase, y función cognitiva sistémica que cualquier artefacto puede cumplir.

\subsection{La función propia y la sistémica en relación con los artefactos cognitivos}

La vasta literatura sobre el concepto de función conduce a un amplio debate que, en gran medida, se desarrolla en conexión con reflexiones de la filosofía de la técnica. Para nuestros propósitos, relacionados con la definición de artefacto cognitivo propuesta más arriba, utilizaremos una distinción conceptual que elabora Beth Preston $(1998,2012)$ a partir de su lectura de este debate (cf. Wrigth, 1973; Cummis 1975; Millikan 1984). Se trata de la distinción analítica entre función propia y función sistémica.

Por un lado, las funciones propias se caracterizan por el hecho de que pueden ser invocadas en el marco de explicaciones evolutivas que dan cuenta de la presencia de un ítem funcional, ya sea un órgano, un artefacto o una parte de un sistema técnico. Desde esta perspectiva, una función propia técnica se entiende como la capacidad que ha tenido un artefacto en la historia evolutiva y que sigue existiendo a partir de la reproducción de dicha función, por parte de agentes intencionales o mecanismos naturales. Pero esta noción también sirve, en el marco de modelos intencionalistas o no-reproductivistas que prescinden de la referencia a una historia reproductiva, para señalar cuál es la capacidad privilegiada por un agente intencional (típicamente el diseñador) entre las múltiples y heterogéneas prestaciones o potencialidades que exhibe un determinado artefacto.

Por otro lado, las funciones sistémicas constituyen aquellas capacidades que tiene un ítem particular en relación a los fines propuestos por un usuario, independientemente de su historia en cuanto linaje técnico. Una silla, por ejemplo, tiene la función propia de servir como objeto para sentarse, pero tiene funciones sistémicas típicas como la de funcionar como escalera para alcanzar objetos que se hallan muy alejados, o funciones menos típicas -pero legítimamente incluibles en la categoría sistémica- como la de trabar una puerta.

En este trabajo no defenderemos una perspectiva sobre el modo en que se establecen las funciones (cf. Parente \& Crelier, 2015), si de manera intencional (por parte de diseńadores o usuarios), o como fruto de una historia de selección (natural o artificial). Nos interesa destacar una diferencia crucial entre ambos tipos de función: la normatividad que caracteriza a las funciones propias frente a 
las sistémicas. Como vimos, la función propia de los artefactos hace referencia o bien a la historia de selección a la cual el artefacto estuvo sujeto (para los reproductivistas), o a los usos privilegiados por el diseñador (para los intencionalistas). En ambos casos, hay usos correctos e incorrectos predeterminados. En el caso de la silla, la mayoría estaría dispuesto a aceptar que ésta tiene la función de permitir que una persona pueda sentarse.

En el caso de la función sistémica no es posible invocar una normatividad semejante, pues estamos ante los usos improvisados que un artefacto pueda desarrollar en determinados entornos naturales o sociales. Por ejemplo, usar una silla para pararse en ella y así alcanzar un objeto que nos sobrepasa en altura o utilizar un destornillador para abrir una lata de pintura. Todos estos son algunos ejemplos de usos idiosincráticos que no estaban contemplados por los diseñadores del artefacto ni por la historia de uso cultural que han tenido dichos artefactos. Así pues, mientras la función propia está vinculada con cierto tipo de normatividad, esto es, cómo debería usarse un artefacto, la función sistémica parece carecer o romper toda norma, pues los usuarios suelen utilizar objetos técnicos de manera no habitual o establecida (Preston, 2012. p, 143).

Ahora bien, ¿cómo se podría aplicar estas reflexiones a los artefactos cognitivos? De acuerdo a nuestro marco explicativo, nos parece más adecuado definir la función propia de un artefacto cognitivo mediante la nota definitoria de que éstos poseen una función propia de naturaleza cognitiva, lo cual requiere algunas especificaciones como las siguientes.

En primer término, se trata de afirmar que la función propia de un artefacto cognitivo es de naturaleza cognitiva en oposición a la posesión de una función propia de naturaleza práctica. Mientras que una calculadora posee la función propia cognitiva de "calcular", una sierra posee la función propia práctica de "aserrar".

En segundo término, es pertinente enfatizar la normatividad ya indicada de las funciones propias. Mientras que la función propia se realiza correcta o incorrectamente (un brújula indica correctamente - o no- los puntos cardinales), la función sistémica no posee una normatividad semejante, pues depende de las prácticas y los intereses ocasionales de los usuarios. Esto trae la siguiente consecuencia: mientras que los artefactos cognitivos poseen funciones propias de naturaleza cognitiva, cualquier artefacto (posea o no funciones propias cognitivas) puede cumplir una función cognitiva en un sistema determinado: la ubicación de los vasos por parte de un barman cumple una función mnemotécnica, aunque la función propia de los vasos no sea cognitiva, sino práctica. Como ya indicamos más arriba, la idea de artefacto cognitivo no se trivializa, pues no cualquier artefacto posee una función propia de esa naturaleza.

En tercer término, la función propiamente cognitiva no se restringe a una sola clase de tareas, sino que puede estar ligada con las variadas capacidades cognitivas 
humanas y no humanas: la memoria, la atención, la percepción, la navegación en el entorno, el cómputo numérico, etc.

En cuarto término, lo dicho hasta aquí no implica que un mismo artefacto pueda tener una sola función cognitiva, pues pueden alegarse ejemplos donde coexisten múltiples funciones cognitivas propias, como es el caso de una computadora (cuya función puede enunciarse en niveles más abstractos y generales, como computar, o en otros más concretos, como procesar textos, calcular, procesar imágenes, registrar sonidos, etc.).

En quinto lugar, tampoco afirmamos que la función cognitiva se encuentre desligada de la función práctica, pues veremos ejemplos prototípicos donde ambas se encuentran entrelazadas, como un GPS o una brújula.

En sexto y último lugar, no se trata de atribuirle indebidamente alguna clase de agencia a los artefactos, pues en realidad la función es realizada por el agente que utiliza el artefacto. Sin embargo, mientras que usualmente resulta claro el rol agencial de quien utiliza el artefacto (un marinero consulta un mapa para llevar adelante con éxito su navegación), a veces resulta lícito extender metafóricamente la idea de agencia, como cuando afirmamos que una computadora computa, una calculadora calcula, etc.

\subsection{Taxonomía gradualista de las funciones cognitivas propias}

La idea de elaborar una taxonomía de los artefactos cognitivos no es nueva. A medida que estos artefactos han ganado interés académico, se han hecho diversas escalas que pretenden clasificarlos a partir de sus propiedades (Brey 2005; Fasoli 2017; Heersmink 2015). En primer lugar, se destaca el esfuerzo de Richard Heersmink quien, a partir de un atento estudio de la tradición filosófica, distinguió dos modos de clasificación de los artefactos cognitivos. Por un lado, el enfoque "cognitivamente-centrado" (cognitive-centered) que clasifica a los artefactos a partir de las capacidades cognitivas asociadas a los artefactos cognitivos, esto es, su ayuda a capacidades tales como la memoria, la percepción, etc. (Brey 2005). Por otro lado, el enfoque "artefactualmente-centrado" (artifact-centered) que, centrándose en el artefacto y no en su proceso cognitivo, clasifica a los artefactos a partir de su estructura, esto es, estructura representacional o no-representacional (ecológica) (Heersmink, 2013, p. 472). Según Heersmink, para un buen entendimiento de los artefactos cognitivos se tiene que descartar el enfoque "cognitivamente-centrado" puesto que se podría caer en el sesgo antropocentrista (Heersmink, 2013, p. 468). Esto se debe a que entender exclusivamente a los artefactos a partir de los procesos cognitivos corre el riesgo de empezar y terminar en el hombre. Una manera de evitar esta suerte de antropocentrismo es clasificar a los artefactos a partir de un enfoque "artefactualmente-centrado". Según el autor, este enfoque promete ser más neutral a la hora de clasificar y entender estos artefactos. 
En segundo lugar, se destaca el esfuerzo de Marco Fasoli quien desarrolla una nueva taxonomía teorética de los artefactos cognitivos basada en las distintas clases de interacciones que tenemos con esta clase de artefactos (Fasoli 2017). Según Fasoli, los anteriores enfoques de clasificación de los artefactos cognitivos caen en el problema según el cual no logran captar diferencias esenciales de estos tipos de artefactos. Particularmente, tanto el enfoque "cognitivamente-centrado" como el "artefactualmente -centrado" son ciegos con respecto a las diferencias en las maneras de cómo estos artefactos cambian nuestras tareas cognitivas (Fasoli, 2017, p. 679). Por su parte, Fasoli desarrolla un nuevo enfoque de clasificación denominado "enfoque interaccionalmente-centrado" (interaction-centered approach) que explora la relación entre artefactos y la mente. De acuerdo con este enfoque, la relación que tenemos con los artefactos puede darse de manera complementaria, sustitutiva o constitutiva. En primer lugar, la relación complementaria propone que el artefacto complementa una tarea cognitiva que estamos llevando a cabo. Piénsese en el mapa o en el compás cuando respectivamente estamos ubicándonos espacialmente o cuando trazamos figuras geométricas; en ambos casos, estos artefactos respectivamente nos complementan actividades cognitivas como la navegación o la proporción. En segundo lugar, la relación sustitutiva propone que el artefacto no substituye nuestros procesos cognitivos, el cual podría existir independientemente. Por ejemplo, los artefactos tales como el navegador GPS o las calculadoras substituyen procesos que podríamos hacer nosotros - con nuestra mente desnuda-, pero que preferimos delegarlo a un artefacto. En tercer lugar, la relación constitutiva propone que el artefacto el artefacto cumple una condición necesaria para ciertas tareas que de otra forma no podrían hacerle prescindiendo de éste. Por ejemplo, los textos. De acuerdo con Fasoli, los textos son artefactos cognitivos constitutivos puesto que la lectura no podría hacerse de otro modo sino es con éstos.

En nuestro caso, la idea de función propia cognitiva nos permite elaborar una taxonomía gradualista según el grado en que esta función se destaca como propia frente a las funciones prácticas. Esta taxonomía podría enmarcarse dentro de lo que podría ser un "enfoque funcionalmente centrado" (function-centered) que nos servirá para investigar la idea de artefactos cognitivos fuera del ámbito humano (en la segunda sección). Si bien esta escala expresa un continuum, en ella podemos distinguir tres momentos diferenciados, que van "de menor a mayor" presencia de la función cognitiva. A partir entonces de esta definición ampliada y la taxonomía propuesta de los artefactos cognitivos, presentamos el siguiente esquema ${ }^{1}$ :

${ }^{1}$ El siguiente esquema fue específicamente diseñado por los autores de este trabajo para presentar de una manera clara las ideas aquí expuestas. 


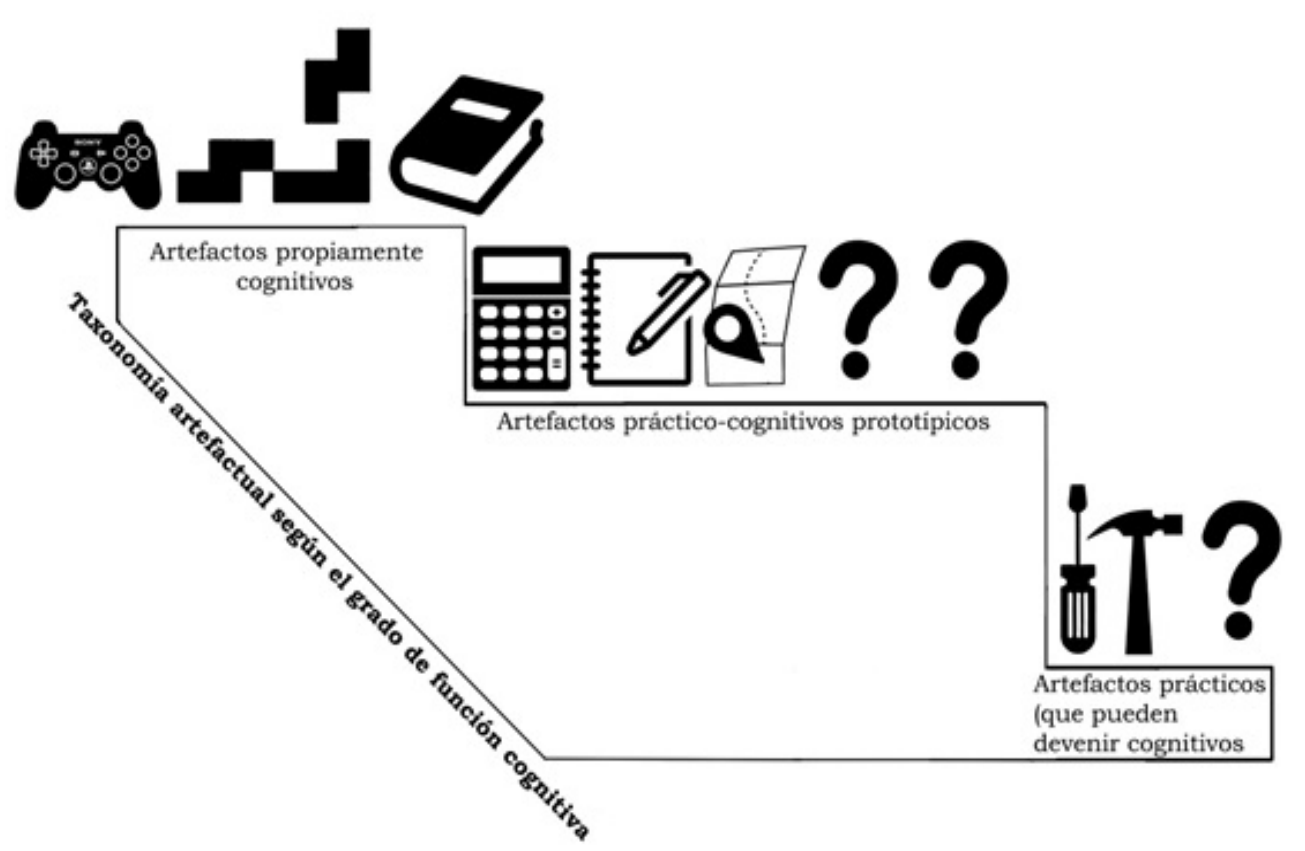

Epígrafe: en el primer peldaño, ubicamos a los artefactos que tienen función propiamente cognitiva que puede desligarse de toda función práctica (videojuegos como el Tetris y libros). En el segundo peldaño, ubicamos a los artefactos que tienen funciones práctico-cognitivas, esto es, ayudan a la cognición a la vez que ayudan a realizar tareas diarias (calculadoras, notebooks, mapas o sistemas GPS, etc). En el tercer peldaño, ubicamos a los artefactos que tienen funciones propias de naturaleza práctica, aunque podrían adquirir funciones cognitivas sistémicas (martillo, destornillador).

(1) En el ápice de esta escala se hallan los artefactos cognitivos cuya función propia cognitiva no se cumple de manera acoplada con funciones prácticas o práctico-cognitivas. Aquí tenemos artefactos que si bien requieren la realización de determinadas acciones en el entorno, estas acciones se subordinan a la función cognitiva misma. En esta dirección, Kirsch y Maglio proponen el ejemplo del videojuego Tetris para ilustrar su noción de acción epistémica, pues allí se aprecia cómo la acción del jugador (en este caso, rotar o trasladar "zoids" en una pantalla) tiene la finalidad de producir una transformación cognitiva (en este caso, resolver el problema de inserción de los zoids que nos plantea este juego) (Maglio and Kirsh 1992). La manipulación de símbolos matemáticos o lingüísticos (mediada a menudo por artefactos técnicos) permite la realización de tareas cognitivas que serían en algunos casos irrealizables sin esa mediación (Kirsch y Maglio 1992, p. 514). En general, la consideración de los sistemas simbólicos como artefactos con función propia de naturaleza cognitiva permite ofrecer una gran variedad de ejemplos, desde la operación con números hasta la lectura de relatos literarios, cuya finalidad principal es transformar nuestra cognición (entendida en un sentido amplio que incluye la emoción y la (re) categorización conceptual). Más 
cercanos a nuestro mundo tecnológico, podemos incluir aquí a los videojuegos en general, que ponen en escena una "realidad virtual" y permiten que las acciones mentales (por ejemplo, los planes mentales de acción) se hagan in extremis antes de cualquier movimiento o incluso que queden confinados en el ámbito de lo mental.

(2) En el medio de esta escala gradual se hallan los artefactos cognitivos "prototípicos", aquellos a los que podemos atribuir con claridad una función propia de naturaleza cognitiva, lo cual sirve incluso para identificarlos. Un sistema de navegación GPS, una libreta de anotaciones o un mapa de trenes tienen la función de facilitar, mejorar o permitir la realización de tareas cognitivas específicas, como orientarse en el espacio, registrar información para un uso futuro o encontrar estaciones de tren cercanas. En estos casos, se advierte la coexistencia de una dimensión práctica en las tareas que estos artefactos nos permiten realizar (trasladarnos a un determinado sitio, planificar un recorrido, recordar una dirección, etc.). Pero su integración en una praxis particular no le quita relevancia a la función cognitiva general, que en estos casos se sigue destacando: resulta más atinado afirmar que la función de un GPS es permitirnos navegar en el espacio que permitirnos regresar al hotel. De hecho, evaluamos el funcionamiento de estos artefactos según la función propia cognitiva que les hemos asignado.

(3) Al final de esta escala gradual se encuentran los artefactos que no poseen un rol funcional en la realización de tareas cognitivas. Existen multitud de artefactos cuyos fines son prácticos y realizan su función produciendo -cuando son utilizados correctamente- determinados cambios en el entorno, como las herramientas del estilo de un martillo, un destornillador o una sierra; u ofreciendo posibilidades de acción que no estaban anteriormente disponibles, como una silla o un florero. Ciertamente, estos artefactos tienen potencialidad para adquirir funciones cognitivas, pero éstas son de carácter sistémico. Cuando colocamos un destornillador en una esquina de la mesa para recordar que debemos usarlo allí, esto no les otorga la normatividad de las funciones propias: no podemos afirmar que el destornillador funcione mal porque no ha cumplido correctamente la mencionada función mnemotécnica.

En estos casos las funciones sistémicas ocasionalmente adquiridas también suelen hallarse desligadas de la praxis más inmediata: todas las mañanas me ejercito haciendo cálculos para mejorar mi rendimiento cognitivo general, o leo un libro para adquirir conocimientos de una época histórica determinada, etc. Ciertamente, el efecto sobre el sistema cognitivo puede tener incidencia en la acción sobre el mundo por parte de los respectivos agentes, pero no hay una relación especificable a priori entre el artefacto y la acción realizada por el agente luego de ser transformado cognitivamente por el artefacto. Esto se aplica ya a los artefactos cognitivos prototípicos: un ingeniero que planifica una construcción edilicia suele utilizar medios artefactuales de diversa índole, desde calculadoras hasta computadoras, pero el uso de un artefacto determinado no guarda una relación con 
la clase de obra en la que el ingeniero se encuentra trabajando. Así, incluso los artefactos cognitivos que usualmente se insertan en tareas prácticas pueden usarse sólo para fines puramente cognitivos, como el uso de herramientas de tracking para el fin de ampliar el conocimiento de los rasgos topográficos del entorno.

\section{Artefactos cognitivos fuera del ámbito humano}

En la sección primera elaboramos una definición de artefacto cognitivo con base en la noción de función propia, lo cual nos permitió elaborar una taxonomía según el grado de función cognitiva en contraposición con las funciones prácticas de los artefactos. Estas herramientas conceptuales nos permiten ahora acercarnos al ámbito no-humano, en una aproximación que será a su vez gradual. En primer término, sostendremos que los animales poseen efectivamente capacidades técnicas, concretamente, que existe un ámbito técnico no-humano de fenómenos de fabricación y uso de herramientas y artefactos. En segundo lugar, sostendremos que puede atribuirse una función cognitiva incipiente a estos artefactos, como es el caso de la ampliación de la percepción facilitada por los palitos termiteros fabricados y usados por chimpancés. Esto nos llevará a profundizar nuestra indagación de las funciones propias cognitivas no-humanas a partir de dos ejemplos, los cuales ilustran la distinción de Heerminsk entre funciones cognitivas representacionales y ecológicas. Según esto, defenderemos la idea de que artefactos como las telas de araña poseen una función cognitiva incipientemente propia y caracterizable como representacional; y que los artefactos como los panales de abeja y los nidos tienen una función de esa clase, pero de naturaleza ecológica, en tanto su locación permite tareas complejas de navegación. Finalmente, aplicaremos la taxonomía gradualista a estos ejemplos no-humanos.

\section{1 ¿Hay técnica no humana?}

La técnica animal es un campo investigativo que no ha recibido la suficiente atención por parte de los estudiosos actuales de la técnica. Así, en la literatura filosófica de la técnica, las reflexiones sobre la capacidad de los animales para crear herramientas ocupan pequeños espacios, excursos o pies de página (Leakey 1994). Esto ha dado pie a que se considere que los animales no tienen una técnica en sentido estricto. Lejos de entrar en ese debate, nos interesa defender al menos la existencia de habilidades técnicas no humanas en cuanto a uso y fabricación de herramientas (tool use \& tool making). Estas habilidades han sido interpretadas de diferente manera, especialmente en relación con la evolución del linaje humano y con la complejidad de la cognición animal (Baber, 2003, 2006; Hansell \& Ruxton, 2008; St Amant \& Horton, 2008).A continuación, repasemos brevemente una parte de la evidencia empírica sobre fabricación de herramientas en animales. 
Una serie de observaciones de cuervos de Nueva Caledonia (Corvus moneduloides) han mostrado que estas aves son capaces de construir herramientas, tanto en los laboratorios como en su estado salvaje (Hunt 1996). Concretamente, los cuervos son capaces de modificar pequeñas ramas para extraer larvas de la corteza de un árbol. Pero, esta clase de habilidades no es una característica exclusiva de los cuervos, sino que abarca a otras aves como los grajos o grajas (corvus frugilegus) (Bird and Emery 2009).

En términos generales, la evidencia sobre esta habilidad técnica se ha hecho extensiva en otras especies, como por ejemplo, los primates. Se ha registrado que algunos primates como los chimpancés africanos (pan troglodytes) y macacos japoneses (macaca fuscata) reconocen la funcionalidad de distintos elementos (tales como piedras, palos, etc.) que utilizan como herramientas (Goodall, 1964). La habilidad de los chimpancés va más allá de ser hábiles constructores. Algunos autores han observado que algunos primates conservan las herramientas que construyen para usarlas posteriormente (Mulcahy and Call 2006a; Osvath 2009; Osvath and Osvath 2008). Casos como los de conservación de herramientas hacen pensar que los primates y, algunas aves como las charas californianas (aphelocoma californica)(Clayton, Bussey, and Dickinson 2003; Clayton and Dickinson 1999; Emery and Clayton 2001), tienen prospección o pensamientos orientados hacia el futuro (foresigth thinking).

Tanto el caso de los cuervos como el caso de los primates demuestran que algunos animales cuentan con niveles de cognición realmente complejos que no pueden ser explicados acudiendo simplemente a las explicaciones genéticas o conductas condicionadas del animal. Esto se debe a que las explicaciones genéticas son limitadas cuando se trata de explicar el uso y la fabricación de herramientas en distintas comunidades de chimpancés de la misma especie (Boesch and Boesch 1990). A su vez, se podría decir que las conductas de uso y fabricación podrían ser respuestas condicionadas por parte de los animales, pero la mayoría de casos fueron observados en el ambiente salvaje.

Estos ejemplos fortalecen la tesis de que los animales fabrican y usan herramientas, con lo cual se advierte la presencia de habilidades físicas o motoras relativamente sofisticadas. ¿Pero, acaso estas destrezas físicas en cuanto a la fabricación y uso de la herramienta podrían involucrar elementos cognitivos? Es decir, ¿podrían estos usos de las herramientas considerarse como usos genuinos de herramientas o artefactos cognitivos?

\subsection{Artefactos animales con funciones cognitivas}

Para responder la pregunta recién formulada, retomemos con algo más de detalle la evidencia de uso de herramientas en primates. Los investigadores Crickette Sanz, Josep Call y David Morgan comprobaron que los chimpancés (pan troglodytes) salvajes del Triángulo de Goualougo (República del Congo) 
son capaces de utilizar herramientas de diversas formas para extraer termitas de un nido (Sanz, Call, \& Morgan, 2009). De acuerdo con las observaciones, los chimpancés utilizaban como herramienta los tallos de una planta conocida como marantaceae. En algunas ocasiones, los chimpancés solamente utilizaban un tallo. En otras ocasiones, los chimpancés utilizaban un tallo duro y otro más laxo para extraer termitas. También se observó que los chimpancés modificaban la herramienta antes usarla. En este caso, las herramientas sufrían pequeñas modificaciones pues los chimpancés mordisqueaban un extremo del tallo para hacer puntas deshilachadas que luego introducían en los nidos para pescar termitas. En los casos en los cuales las herramientas no eran modificadas, los chimpancés la utilizaban de diversas formas. Además de ser utilizada para pescar termitas, las herramientas eran utilizadas para pinchar los nidos y así crear nuevos túneles para extraer más termitas (p.3).

Tal como afirman algunos autores, el uso de la herramienta por parte de los chimpancés implica planes de acción (Byrne, Sanz, \& Morgan, 2013). Debido al nivel de complejidad física requerida, el uso de la herramienta cuenta con una organización jerárquica dividida en rutinas y subrutinas (pp. 57). Por ejemplo, en el caso de los chimpancés de Goualougo, la rutina de uso de herramienta consiste en pescar termitas con herramientas. Esta rutina se divide en sub-rutinas como puede ser pinchar los túneles ya hechos para atraer a las termitas u oler la herramienta para saber si hay termitas en el nido. Al detectar cierto tipo de hormonas que desprenden este tipo de termitas, el chimpancé seguirá picando el túnel hasta hallar su alimento. De lo contrario, éste abandonará este nido y se irá a buscar otro donde efectivamente halla termitas.

La conclusión a la que queremos llegar en este apartado es que los animales son capaces de utilizar herramientas en tareas cognitivas complejas. En el marco de estas últimas, los artefactos y herramientas fabricados por animales como los chimpancés cumplen un papel central. Si aceptamos que la interpretación de Byrne y compañía en cuanto a los planes de acción es correcto, entonces el uso y fabricación de herramientas podría tener un uso cognitivo incipiente. Esto se debe a que por medio del palito termitero, el chimpancé está extendiendo parte de su sistema perceptual. Particularmente, el chimpancé está extendiendo su olfato y, por qué no, su visión y su tacto, debido a la incapacidad para ver y meter su malo a través de los pequeños túneles, aunque esta función cognitiva puede considerarse aún incipiente, pues prima la habilidad física, motriz y coordinativa del animal.

En lo que sigue, defenderemos que la función cognitiva incipiente que hemos destacado puede considerarse también una función propia, en el sentido defendido en la primera sección. El ejemplo de los panales y de las telarańas nos servirán para mostrar dos clases de funciones cognitivas propias, una de naturaleza ecológica y otra de naturaleza representacional. 


\subsubsection{El caso de los nidos y panales: función propia cognitiva ecológica}

Autores como Peter Carruthers se han centrado en el caso de los insectos navegantes (abejas, avispas, etc.) y han afirmado que las abejas cuentan con estructuras psicológicas basadas en planes que envuelven tanto deseos como creencias (Carruthers 2006). Particularmente, este autor afirma que las abejas tienen un sistema de generación de información que construye representaciones relativas a la dirección y las distancias entre los objetos (flores, etc) y el nido (p., 286). En este sentido, James L. Gould ha discutido el caso de la construcción del nido en relación con las marcas ambientales. Gould ha afirmado que las abejas, a través de una serie de marcas ambientales, pueden desarrollar cierto tipo de "mapas mentales" que le ayudan a orientarse en su entorno (Gould 1986). Gallistel describe del siguiente modo cómo la percepción visual de los panales sirve -según él- para la formación de un "mapa cognitivo":

The discovery that the wasp locates her nest by reference to the landmarks or terrain features that surround it implies that she notes the position of her nest relative to these landmarks before her departure, and, in fact, it has since been shown that the departing wasp makes a special survey flight for that purpose (Zeil, 1993a). Moreover, it implies that when she returns she uses the remembered position of her nest relative to those landmarks to guide her flight path to a landing site. A record of the position of one or more points of interest relative to the positions of landmarks or salient terrain features is a map. A map that resides in the brain of an animal rather than on a sheet of paper or in the memory banks of a computer is called a cognitive map. Thus, the results of Tinbergen and Kruyt's experiment suggest that the wasp makes a cognitive map of the immediate vicinity of her nest and uses that map to choose a landing site (Gallistel 1998).

En el caso de las aves, puede afirmarse que existen habilidades cognitivas semejantes, y que en ellas los nidos cumplen también una función cognitiva como puntos de referencia para los diversos modos de orientación en el entorno (cf. McMillan et. al. 2015). Ciertamente, los estudios sobre los métodos usados por las aves para orientarse hacia el nido toman mayormente en cuenta parámetros como el campo magnético o la ubicación de las estrellas, sin que la ubicación del nido sea particularmente destacada. Sin embargo, se asume por ejemplo que el objetivo de la conducta de regreso al hogar (homing behavior) tiene como referencia el nido, al menos en aquellas especies que fabrican nidos y no regresan, por ejemplo, a un aviario construido por humanos.

Como resulta entonces evidente, los nidos y panales son artefactos fabricados por diversas especies no humanas que cumplen una función esencial en la tarea práctico-cognitiva de orientarse en el entorno. Estas especies construyen, ubican e identifican nidos y panales, lo cual les permite a su vez construir, según la interpretación de algunos autores, mapas mentales que representan una parte 
del entorno, que a su vez se insertan en tareas prácticas como regresar al hogar o buscar alimento ${ }^{2}$.

Ahora bien, la función práctico-cognitiva de estos artefactos no se realiza en principio con base a su estructura representacional, como sí sucede con un mapa físico o mental del terreno. Como hemos visto, algunos autores piensan que las aves y abejas construyen representaciones del entorno utilizando la locación de estos artefactos, pero nos interesa destacar que la locación de los mismos posee una relevancia cognitiva, lo cual sucede en cierto modo como algo previo (e incluso en ocasiones independiente) de su uso representacional. Esto se aviene a la idea introducida por Heerminsk que comentamos más arriba, según la cual existen artefactos cognitivos de naturaleza ecológica, que permiten o favorecen la realización de tareas cognitivas sin representar ellos mismos objetos o eventos. Esta clase de artefactos permiten que el agente codifique información relevante de manera más sencilla o favorable a sus intereses prácticos. El agente ha codificado allí cierto tipo de información a través de su referencia espacial y su locación (Heersmink 2013). Los ejemplos en humanos incluyen la acción de dejar las llaves en un sitio determinado para que no sea necesario recordar dónde se encuentran, u organizar el espacio de trabajo de manera que la ubicación de los objetos facilite la tarea que uno está haciendo (Kirsh 1995).

Nuestra inclusión de los nidos y los panales en la categoría de los artefactos cognitivos ecológicos se enfrenta a la siguiente objeción. Se puede alegar que estamos ampliando la categoría de artefacto cognitivo hasta volverla trivial. Cualquier elemento del entorno, sea o no un producto intencionalmente diseñado para tal fin, permite que un organismo codifique representacionalmente información sobre el entorno en el que habita. Así, el sol mismo podría entenderse como un artefacto cognitivo ecológico, en tanto las abejas lo utilizan para calcular sus vuelos hacia y desde el panal, expresándolo además en las danzas que realizan para transmitir la ubicación de esos sitios. En suma, cualquier elemento del entorno puede entenderse como un "artefacto cognitivo", pues basta que sea "adaptable" o, como mínimo, que el organismo lo adopte para sus fines representacionales y prácticos.

Para responder a esta objeción regresaremos a la distinción entre función sistémica y función propia. Recordemos que la función sistémica se relaciona con las capacidades que tiene un ítem particular en cumplir una función contingentemente asignada por el agente, y la función propia representa en cambio una

\footnotetext{
${ }^{2}$ Adviértase que existen dos interpretaciones posibles del uso cognitivo de los panales o nidos. Según la primera interpretación, afín a la idea de una mente extendida, se trata de ítems que debemos considerar como parte de la mente animal, como el cuaderno de Otto en el célebre ejemplo de Clark y Chalmers (1998). Según la segunda interpretación, más cercana a la de Gallistel, se trata de insumos o inputs que la mente animal utiliza para construir representaciones del entorno. La idea de artefactos cognitivos propuesta en este trabajo no precisa adherir a ninguna de estas dos posturas, aunque ha manifestado simpatía por la idea de una mente extendida.
} 
capacidad privilegiada y predeterminada por la historia evolutiva o por el diseño, que puede ser realizada correcta o incorrectamente. Aquí resulta interesante advertir los ejemplos propuestos por Heerminsk para ilustrar la idea de función ecológica suelen referirse a funciones sistémicas, como la ubicación de objetos en sitios especiales o una organización contingente del espacio para facilitar un plan de acción. Es posible agregar un sinfín de ejemplos de esta naturaleza: las migas para marcar el camino de regreso hogar que Hansel y Gretel ubican en el cuento de los hermanos Grimm, los vasos que el barman ordena para recordar los ingredientes de los tragos que ha de preparar, etc. Aunque también hay ejemplos no sistémicos: poner un logotipo en un cartel indicando la presencia de un comercio cumple una función cognitiva ecológica no meramente sistémica, pues puede realizarla correcta o incorrectamente (por ejemplo si el cartel es desplazado de lugar).

En el caso no humano, la ubicación de los nidos y de los panales cumple funciones ecológicas que pueden considerarse como incipientemente propias. En efecto, es de suponer que la historia evolutiva los ha seleccionado, al menos en parte, debido a que permiten la realización de tareas práctico-cognitivas como las mencionadas, que pueden además cumplir mejor o peor. Por ejemplo: si un ave construye un nido que se confunde con otro elemento del entorno, la conducta de regreso al hogar se verá dificultada; o si las abejas construyen un panal en un sitio instable, de modo que éste se desplaza espacialmente, no podrán orientarse eficientemente a partir de esa referencia ecológica.

Finalmente, en tanto se vislumbra una función cognitiva propia, podemos ubicar esta clase de artefactos ecológicos en el ámbito de los artefactos cognitivos prototípicos. Resta discutir si el carácter meramente incipiente de la función propia los distingue de los correspondientes artefactos humanos. Pero es suficiente para nuestro propósito defender provisionalmente dicha ubicación conceptual.

\subsubsection{El caso de las telas de arańa: función propia cognitiva representacional}

Recientes estudios en cognición de arácnidos sugieren que las arañas descargan tareas cognitivas en sus telarañas, lo cual las convertiría en una de las primeras especies con una cognición que no está confinada completamente dentro de su cabeza. En el año 2008, el investigador Hilton Japyassú recolectó 20 especies pertenecientes a diversas familias de arácnidos por todo el Brasil con el objetivo de comparar las prácticas de caza estereotipadas versus las nuevas prácticas de caza que pueden darse de manera innovadora (Penna-Gonçalves, Garcia \& Japyassú, 2008). Según el estudio realizado, se intervinieron las telarañas producidas por tres familias de arácnidos (Uloboridae, Araneidae y Tetragnathidae) con el objetivo de comparar su capacidad de plasticidad comportamental, esto es, la capacidad de ejecutar nuevos comportamientos ante la presión de distintos procesos ecológicos; en este caso, la modificación de la telarańa. 
Los investigadores tomaron especies como tetragnátidos (Tetragnathidae) y ulobóridos (Uloboridae); arañas conocidas por tejer sus redes en forma de pequeñas órbitas (orbweb) que se abren en pocos radios y espirales. Luego, ellos compararon la forma de tejer y cazar de estas especies con la forma en que los terídidos (Theridiidae) fabrican su telarańa y cazan. Los terídidos son especies que fabrican telarańas que no siguen una forma regular órbitocircular (orbweb) como frecuentemente se encuentra en los tetragnátidos, sino que su telaraña (cobweb) está construida siguiendo formas irregulares y tridimensionales que se extienden hasta el piso conformando 'hilos de captura' (gumfoot) en los cuales se quedan atrapadas las presas (Allana 2014). A partir de su telaraña característica (cobweb), los terídidos han desarrollado una forma de cazar que consiste en atrapar a la presa abalanzándose sobre ella (reeling behaviour) una vez que han sentido la vibración por medio de los hilos de captura.

En un experimento los investigadores modificaron la telaraña de los tetragnátidos con el fin de crear hilos de captura artificiales (pseudogumfoot) en los cuales se quedaron atrapadas algunas de las presas (por ej., pequeñas polillas). Con la modificación de su telaraña, los tetragnátidos se verían obligados a cambiar sus tácticas de caza por la forma en que los terídidos (Theridiidae) cazan. Los resultados del experimento fue que la especie Zosis geniculata, perteneciente a la familia de los ulobóridos, modificó su comportamiento con respecto a la telarańa intervenida. Esto quiere decir que la Z. Geniculata, una especie que nunca antes usó la táctica de los hilos de captura y balanceo, modificó su comportamiento de tal forma que cambió sus prácticas de caza. Una vez que la telaraña fue modificada, la araña se abalanzó sobre la presa una vez que sintió su presencia por medio de los hilos artificiales dispuestos por los investigadores (Penna-Gonçalves, Garcia, \& Japyassú, 2008, p. 357).

La plasticidad del comportamiento de las especies analizadas suscitó diversas preguntas a propósito de cómo era posible que las arańas modificaran su comportamiento ante problemas con los cuales quizá nunca antes se habían topado. Sobre todo, cuando las arańas tradicionalmente se han considerado como especies que tienen un comportamiento rígido y estereotipado (Eberhard 1982). La pregunta entonces formulada era sobre la capacidad que estas arańas tienen de archivar información. Por tal razón, el biólogo brasileño Japyassu y el biólogo británico Kevin Laland plantearon la hipótesis según la cual la información acerca del entorno no está depositada únicamente dentro del cuerpo de los arácnidos, sino que dicha información parece extenderse a través de la telarańa (Japyassú \& Laland 2017). Japyassu y Laland proponen la hipótesis según la cual la telaraña actúa como una extensión de los órganos perceptivos de las arańas, pues éstas aumentan la sensibilidad a través de la amplificación o atenuación de vibraciones particulares que les permite detectar movimientos de sus presas a cierta distancia.

La hipótesis de la telarańa como un sistema perceptual extendido afirma que las arañas son capaces de controlar el flujo de información ambiental a través de 
su telaraña, de tal forma que las arañas constantemente están focalizando su atención a ciertas partes de la red. Por ejemplo, algunos estudios de caso centrados en las tácticas de caza de algunas arañas muestran que éstas fabrican sus telarañas más tensas cuando tienen más hambre. Las telarañas tensionadas, a diferencia de las telarañas más flojas, son más receptivas ante los estímulos pues la resonancia de la vibración de la presas se transmite más rápidamente por toda la red (Nakata 2010). De tal forma, las arañas son capaces de ajustar las propiedades de su telaraña dependiendo del grado de atención o información que requieran para cazar. En este sentido, algunas alteraciones de la telarańa por parte de los investigadores hace que una araña tenga más atención focalizada en algunas partes de su telaraña y en otras partes no (Nakata 2010).

Estas investigaciones nos hacen regresar al eje de nuestro trabajo mediante la siguiente pregunta: ¿Es la telarańa acaso un artefacto cognitivo? Consideramos que la respuesta a esta pregunta es afirmativa, en tanto se puede plantear la hipótesis de que la telarańa es una herramienta fabricada por un organismo, que extendería su campo perceptivo. Pero vale aclarar que cuando afirmamos que la telaraña es un artefacto cognitivo estamos pensando en que su función es práctica-cognitiva. Si bien la telaraña cumple con una función cognitiva de procesamiento de la información, esta función no puede ser separada de su función de atrapar presas. De esta forma, la telarańa, como ejemplo de un artefacto cognitivo animal, podría ser considerada como un artefacto que tiene una función cognitiva prototípica, esto es, su función se iguala a la función práctico-cognitiva de artefactos humanos como la calculadora o el mapa debido a que estos artefactos permiten, ya bien sea, recordar información relevante o guiarse en el espacio $^{3}$. Esta función es además propia, en tanto puede suponerse que ha sido

${ }^{3}$ Podría objetarse que la telarańa no es un artefacto en sentido estricto puesto que no cumple con los criterios de uso de herramientas (tool use) propuesta por la tradición (cf. Hansell \& Ruxton, 2008). Según ésta, un objeto puede ser considerado como un artefacto o herramienta si éste: (i) no hace parte del animal, (ii) no está unido al medio ambiente y (iii) puede ser manipulado para lograr algún resultado beneficioso (p. 73). En este sentido, las telarańas orbitocirculares (orbweb) de las especie tetragnátidos y ulobóridos no podrían ser consideradas como herramientas porque i) son producidas por los ganglios productores de seda y, se podría decir que la telarańa hace parte del animal; (ii) las telarañas, al ser dispuestas en lugares determinados, se podría considerar que están sujetas al medio ambiente; (iii) como a primera vista algunas arańas una vez construida la telaraña esperan pacientemente a las presas, se podría decir que la telaraña no es manipulable para lograr determinados objetivos. Pero incluso si se mantienen estos criterios, existen ejemplos que evitan la objeción. Por ejemplo, la especie araneomorfa del género mastophora (Mastophora hutchinsoni) destaca por el hecho de construir una bola pegajosa al final de un extenso hilo que utiliza su telarańa como boleadora para impactar las presas capturándolas, de modo que no viola los mencionados criterios. El otro ejemplo proviene de la especie conocida como "araña con cara de ogro" (Dinopis longipes) cuya táctica de caza consiste en fabricar una pequeña telaraña que sostiene en patas para capturar a modo de atarraya arrojadiza a sus presas (Robinson and Robinson, 1971). A pesar que su telarańa es una secreción, ésta no hace integral del arácnido; asimismo, la telarańa no está unida al medio ambiente pues se caracteriza por el hecho de ser arrojadiza (de hecho, las telarañas de otras especies órbitocirculares tampoco están definitivamente ligadas al 
seleccionada por un largo proceso evolutivo, de modo que pueden hacerse juicios normativos sobre el cumplimiento correcto o inadecuado.

Finalmente, la función de la telarańa puede encuadrarse tanto en la idea de que estos organismos extiende su cognición mediante determinados artefactos, como con la de que fabrican artefactos que poseen una relación representacional con el entorno. Según esta última interpretación, la telaraña sería un signo que, al modo de un índice, está relacionado con la presencia de la presa de manera causal. Si bien ambas interpretaciones suponen la presencia de artefactos que poseen una función propia de naturaleza cognitiva, la segunda interpretación se adecua a la clasificación de Heerminsk, según la cual muchos artefactos cognitivos poseen una estructura representacional. De esta forma, completamos nuestra escala planteada anteriormente en la página nueve de este trabajo. Así, incorporaremos las propuestas de nuestra segunda sección a la escala gradual anteriormente esbozada, lo cual servirá como una síntesis visual de algunas de nuestras ideas principales:

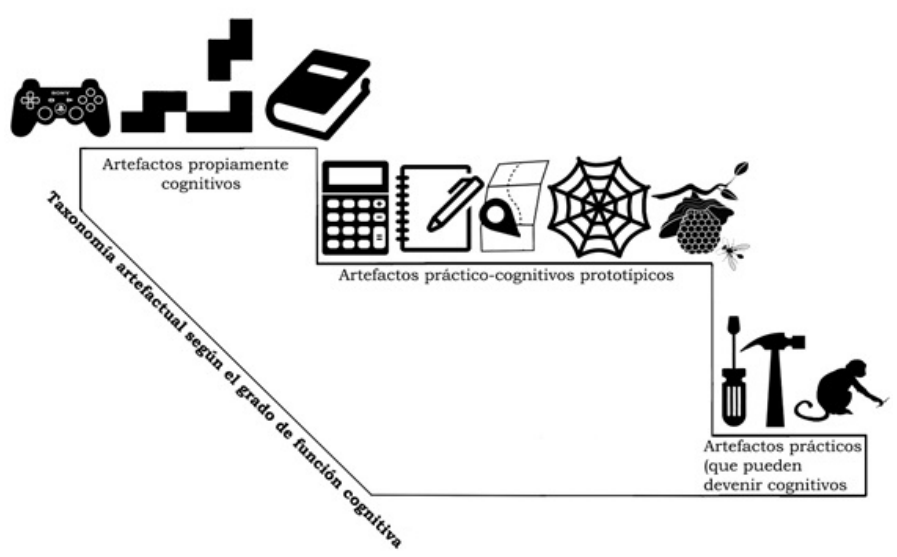

Epígrafe: la presente imagen condensa nuestra escala taxonómica de artefactos cognitivos. Tal como presentamos anteriormente, se ha dividido en peldańos en los cuales aparecen artefactos con función cognitiva o practico-cognitiva. La particularidad de dicha escala es que incluimos, en el tercer peldaño, a los artefactos que tienen funciones propias de naturaleza práctica, aunque podrían adquirir funciones cognitivas sistémicas (martillo, destornillador). En este sentido, decidimos incluir de manera tentativa a los artefactos utilizados por algunos chimpancés como muestra de dicha función cognitiva.

Pero, a primera vista, esta aproximación podría presentarse la objeción acerca de la proximidad filogenética del caso anterior. Específicamente, la disparidad anatómica entre homínidos y arácnidos. Así las cosas, ¿̇será acaso posible hallar

entorno); finalmente, la telaraña puede ser manipulable/modificable para lograr un objetivo, en este caso, atrapar a la presa y de esta forma obtener alimento. Así, pues, estos ejemplos sirven como respuesta a una posible objeción a propósito de la consideración de la telaraña como artefacto cognitivo. 
un artefacto cognitivo más próximos, filogenéticamente hablando, a nosotros? A continuación, como mera sugerencia, revisaremos un estudio en metacognición que parece indicar afirmativamente la posibilidad efectiva de hallar artefactos cognitivos en el mundo animal.

\subsection{3 ¿El caso más próximo? Artefactos y metacognición en chimpancés}

Una serie de recientes estudios experimentales sugieren que los animales tienen capacidades metacognitivas (Foote \& Crystal, 2007; Hampton, 2001; Smith, 2005; Smith, Shields, Schull, \& Washburn, 1997). Así, los experimentos de Smith (2009) indican que los monos rhesus (Macaca mulatta) y los delfines nariz de botella (Tursiops truncatus) son conscientes de sus propios estados de incertidumbre (en el reconocimiento de patrones visuales). Lejos de entrar en el debate correspondiente (Proust, 2015c; Carruthers, 2009; cf. Arango-Muñoz, 2011), nos interesa señalar que algunos de estos estudios parecen apoyar además la idea de que los animales combinan la habilidad de resolver tareas metacognitivas (en la búsqueda de información) con el uso de herramientas. De esta forma, estaríamos ante un caso de uso de herramientas cognitivas.

En este sentido, Perdue y colegas argumentan que ciertas capacidades metacognitivas como la búsqueda de información (information-seeking task), monitoreo y control de procesos se pueden conectar con las dinámicas de uso de herramientas. Estos investigadores hicieron un conjunto de pruebas con chimpancés que debían obtener una recompensa por medio de la utilización de una herramienta (Perdue, Evans \& Beran, 2018). La dinámica inicial consistía en que el chimpancé tenía que utilizar la herramienta para levantar una tapa, en una estación con cinco cubiertas, con el fin de averiguar si allí se había depositado su recompensa o, si por el contrario, estaba vacía. Los chimpancés tenían tres condiciones en las pruebas: veían la recompensa, no la veían, o debían inferir dónde se ubicaba ${ }^{4}$.

${ }^{4}$ La presente imagen fue tomada del texto de Perdue y colegas. Con más detalle, véase: (Perdue, Evans \& Beran, 2018). 


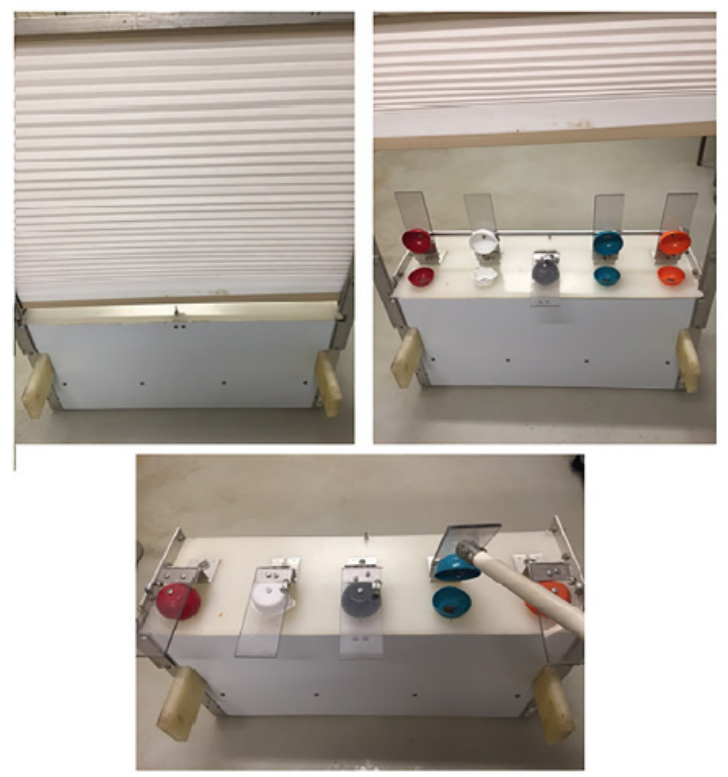

Epígrafe: en las fotografías puede apreciarse la estación que se cubre con una puerta metálica corrediza. Cuando ésta es desatapada, el sujeto puesto a prueba puede acceder a las cinco cubiertas sólo a través del palo. Tal como se precisó anteriormente, los sujetos puestos a prueba podían, en algunas ocasiones, ver previamente dónde se escondía su recompensa.

Posteriormente, los investigadores variaron la prueba buscando respuestas flexibles en donde los chimpancés decidían si querían o no utilizar la herramienta en concordancia a la información que tenían. Los resultados de esta fase mostraron que los chimpancés recurrían a la herramienta cuando no venían ni sabían dónde estaba su recompensa. Así, en los casos en los cuales no se les mostraba dónde había sido escondida su recompensa, los chimpancés tomaban la herramienta que les proveía de información adicional hasta que ellos encontraban la ubicación del alimento y, devolviendo la herramienta al investigador, señalaban la ubicación exacta de su recompensa. En otras ocasiones, tomaban la herramienta cuando no estaban seguros dónde estaba ubicada su recompensa. Esto sugiere que los chimpancés contrastaron la información que se les otorgaba con la información que ellos podían recuperar cuando utilizaban la herramienta.

¿Podrían entonces las herramientas utilizadas por los animales considerarse genuinos artefactos cognitivos? Ciertamente, antes de responder a esta pregunta debemos preguntarnos el rol preciso de los artefactos o las herramientas en estas pruebas. Particularmente, si efectivamente el hecho de recuperar información está ligado al hecho de usar la herramienta o, si por el contrario, ambas cualidades pueden ser disociadas. Pero la evidencia inicial parece avalar la idea de artefactos cognitivos en estos primates cercanamente emparentados al hombre. 


\section{Consideraciones finales}

En este trabajo hemos defendido la idea de que existen animales no-humanos que fabrican artefactos, y que algunos de estos artefactos poseen una incipiente función propia de naturaleza cognitiva. En la primera sección, desarrollamos la idea de artefacto cognitivo con base a la noción de función propia, y propusimos una escala según el grado de presencia de la función cognitiva en contraste con las funciones prácticas. En la segunda sección, abordamos el ámbito no-humano, defendiendo ante todo la idea de que existen fenómenos de fabricación y uso de herramientas en diversas especies. Con ello llegamos a nuestra tesis central consistente en señalar que algunos de estos artefactos poseen una incipiente función propia de naturaleza cognitiva, algo que ilustramos con los ejemplos de las telas de arańa y los nidos y panales.

Si bien consideramos que estos ejemplos parecen ser casos muy sugestivos de artefactos cognitivos por fuera del ámbito humano, consideramos que estos son apenas aproximaciones para encontrar uso y fabricación de artefactos cognitivos en el mundo animal. Si bien el caso de las telarańas y los panales son sugestivos, consideramos que por las distancias filogenéticas estos casos podrían parecer algo forzados, y que sería bueno explorar con detenimiento su presencia en animales emparentados con el hombre, algo que sólo sugerimos brevemente respecto de los chimpancés.

En suma, el caso de los artefactos cognitivos apenas esbozado en este trabajo puede servir como un adelanto positivo en la materia. Pero, por motivos de espacio, dicha línea será desarrollada en otra entrega. Por lo pronto, concluimos que es posible hablar de artefactos cognitivos en animales sólo de manera incipiente.

\section{Referencias bibliográficas}

Arango-Muñoz, Santiago (2011). Two Levels of Metacognition. Philosophia, 39(1), $71-82$.

Baber, Christopher (2003). Cognition and Tool Use: Forms of Engagement in Human and Animal Use of Tools. London: Taylor and Francis.

Baber, Christopher (2006). Cognitive Aspects of Tool Use. Applied Ergonomics, 37(1 SPEC. ISS.), 3-15.

Badallo, Ana Cuevas (2016). Artefactualidad animal. Ludus Vitalis, 24 (45), 155-174.

Bird, Christopher D. and Nathan John Emery (2009). Report Rooks Use Stones to Raise the Water Level to Reach a Floating Worm. Biology, 19, 1410-14.

Boesch, Chiristophe and Hedwige Boesch (1990). Tool Use and Tool Making in Wild Chimpanzees. Folia Primatologica, 54(1-2), 86-99.

Borgo, Stefano, Noemi Spagnoletti, Laure Vieu, Elisabetta Visalberghi, Stefano Borgo, Noemi Spagnoletti, Laure Vieu, Elisabetta Visalberghi Artifact, and Artifact

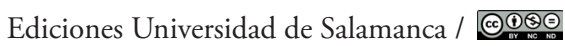

ArtefaCToS, Vol. 8, No. 2 (2019), 2. ${ }^{\text {Época, 27-52 }}$ 
Catego (2013). Artifact and Artifact Categorization: Comparing Humans and Capuchin Monkeys. Review of Philosophy and Psychology, 4(3), 375-89.

Brey, Philip (2005). The Epistemology and Ontology of Human-Computer Interaction. Minds and Machines, 15(3-4), 383-98.

Byrne, Richard W., Crickette M. Sanz, and David B. Morgan (2013). Chimpanzees Plan Their Tool Use. En: C. M. Sanz, J. Call, and C. Boesch (Eds.), Tool Use in Animals: Cognition and Ecology. Cambridge: Cambridge University Press.

Carruthers, Peter (2006). Invertebrate Minds: A Challenge for Ethical Theory. Journal of Ethics, 11(3), 275-97.

Carruthers, Peter (2009). How We Know Our Own Minds: The Relationship between Mindreading and Metacognition. The Behavioral and Brain Sciences, 32(2), 121138; discussion 138-182.

Clark, Andy (2006). Material Symbols. Philosophical Psychology, 19(3), 291-307.

Clark, Andy and David J. Chalmers (1998). The Extended Mind. Analysis, 58(1), 7-19.

Clartcey, William J. (2009). Scientific Antecedents of Situated Cognition. In P. Robbins and M. Aydede (Eds.), The Cambridge handbook of situated cognition. Cambridge: Cambridge University Press.

Clayton, Nicola S. and Anthony Dickinson (1999). Scrub Jays (Aphelocoma Coerulescens) Remember the Relative Time of Caching as Well as the Location and Content of Their Caches. Journal of Comparative Psychology, 113(4), 403-16.

Clayton, Nicola S., Timothy J. Bussey, and Anthony Dickinson (2003). Can Animals Recall the Past and Plan for the Future? Nature Reviews. Neuroscience, 4(8), 68591.

Cummins, Robert (1975). Functional Analysis. The Journal of Philosophy, 72(10), 741764.

Donald, Merlin (2010). The Exographic Revolution: Neuropsychological Sequaelae. In: Lambros Malafouris \& Colin Renfrew (Eds.), The Cognitive Life of Things. Recasting the Boundaries of the Mind (pp. 71-79). Cambridge: McDonald Institute for Archaeological Research, University of Cambridge.

Eberhard, William G. (1982). Behavioral Characters for the Higher Classification of Orb-Weaving Spiders. Evolution, 36(5), 1067-95.

Emery, N. J. and Clayton, N. S. (2001). Effects of Experience and Social Context on Prospective Caching Strategies by Scrub Jays. Nature, 414(6862), 443-46.

Fasoli, Marco (2017). Substitutive, Complementary and Constitutive Cognitive Artifacts: Developing an Interaction-Centered Approach. Review of Philosophy and Psychology, 9(3), 671-87.

Foote, Allison L. and Jonathon D. Crystal (2007). Metacognition in the Rat. Current Biology, 17(6), 551-55. 
Gallistel, Randy (1998). Insect Navigation: Brains as Symbol-Processing Organs. An Invitation to Cognitive Science, 4, 1-52.

Goodall, Jane (1964). Tool-Using and Aimed Throwing in a Community of Free-Living Chimpanzees. Nature, 201 (4926), 1264-1266.

Gould, J. L. (1986). The Locale Map of Honeybees: Do Insects Have Cognitive Maps? Science, 232(4752), 861-863.

Hampton, Robert (2001). Rhesus Monkeys Know When They Remember. Proceedings of the National Academy of Sciences of the United States of America, 98(9), 5359-62.

Hansell, Mike and Graeme D. Ruxton (2008). Setting Tool Use within the Context of Animal Construction Behaviour. Trends in Ecology and Evolution, 23(2), 73-78.

Heersmink, Richard (2012). The Varieties of Situated Cognitive Systems: Embodied Agents, Cognitive Artifacts, and Scientific Practice. Tesis doctoral obtenida de: https://www.google.com/search?client=safari\&rls=en\&q=Heersmink, + Richar$\mathrm{d}+(2012)$. + The + Varieties + of + Situated +Cognitive+Systems:+Embodied +Agents, + Cognitive + Artifacts, + and + Scientific + Practice $\& i e=U T F-8 \& o e=U T F-8$

Heersmink, Richard (2013). A Taxonomy of Cognitive Artifacts: Function, Information, and Categories. Review of Philosophy and Psychology, 4(3), 465-81.

Heersmink, Richard (2015). Dimensions of Integration in Embedded and Extended Cognitive Systems. Phenomenology and the Cognitive Sciences, 14(3), 577-598.

Heersmink, Richard (2016). The Metaphysics of Cognitive Artifacts. Philosophical Explorations, 19, 78-93.

Heersmink, Richard (2018). A Virtue Epistemology of the Internet: Search Engines, Intellectual Virtues and Education. Social Epistemology, 32(1), 1-12.

Hollan, J. D. James, Edwin Hutchins, and David Kirsh (2000). Distributed Cognition: Toward a New Foundation for Human-Computer Interaction Research. ACM Transactions on Computer-Human Interaction, 7(2), 174-96.

Hunt, G. R. (1996). Manufacture and Use of Hook-Tools by New Caledonian Crows. Nature, 379(6562), 249-51.

Japyassú, Hilton F. and Kevin N. Laland (2017). Extended Spider Cognition. Animal Cognition, 20(3), 375-95.

Kirsh, David (1995). The Intelligent Use of Space. Artificial Intelligence. Artificial Intelligence, 73(1-2), 31-68.

Leakey, Richard (1994). The Origin of the Humankind. New York: Basic Books.

Lomas, Derek (2007). Cognitive Artifacts. Proceedings of the 6th ACM SIGCHI Conference on Creativity \& Cognition, 289.

Maglio, Paul and David Kirsh (1992). Some Epistemic Benefits of Action: Tetris, a Case Study. Proceedings of the Fourteenth Annual Conference of the Cognitive Science Society, 14, 224. 
Millikan, Ruth Garrett (1984). Language, Thought, and Other Biological Categories: New Foundations for Realism. The MIT Press.

Mulcahy, N. J. and J. Call (2006). Apes Save Tools Fur Future Use. Science, 312(2006), 1038-40.

Nakata, K. (2010). Attention Focusing in a Sit-and-Wait Forager: A Spider Controls Its Prey-Detection Ability in Different Web Sectors by Adjusting Thread Tension. Proceedings of the Royal Society B: Biological Sciences, 277(1678), 29-33.

Norman, Donald A and Carroll, M. (1991). Cognitive Artifacts. Designing Interaction, Psychology at the human-computer interface, 1, 17-38.

Norman, Donald A. (1992). Design Principles for Cognitive Artifacts. Research in Engineering Design, 4(1), 43-50.

Osvath, Mathias (2009). Spontaneous Planning for Future Stone Throwing by a Male Chimpanzee. Current Biology, 19(5), 190-91.

Osvath, Mathias and Helena Osvath (2008). Chimpanzee (Pan Troglodytes) and Orangutan (Pongo Abelii) Forethought: Self-Control and Pre-Experience in the Face of Future Tool Use. Animal Cognition, 11(4), 661-74.

Parente, Diego y Andrés Crelier (2015). La naturaleza de los artefactos: intenciones y funciones en la cultura material. Buenos Aires: Prometeo.

Penna-Gonçalves, V., C. R. M. Garcia, and H. F. Japyassú (2008). Homology in a Context Dependent Predatory Behavior in Spiders (Araneae). Journal of Arachnology, 36(2), 352-59.

Perdue, Bonnie M., Theodore A. Evans, Michael J. Beran (2018). Chimpanzees Show Some Evidence of Selectively Acquiring Information by Using Tools, Making Inferences, and Evaluating Possible Outcomes. PLoS ONE, 13(4), 1-20.

Preston, Beth (1998a). Why Is a Wing Like a Spoon? A Pluralist Theory of Function. Journal of Philosophy, 95(5), 215-54.

Preston, Beth (1998b). Cognition and tool use. Mind \& Language, 13(4), 513-547.

Preston, Beth (2012). A Philosophy of Material Culture. Routledge.

Proust, Joëlle (2015). The Representational Structure of Feelings. Open MIND, 31, $1-26$.

Robbins, P., Aydede, M. (2009). A Short Primer on Situated Cognition. In P. Robbins, and Aydede, M., The Cambridge handbook of situated cognition (pp. 3-10). Cambridge: Cambridge University Press.

Robinson, Michael H., Robinson, Barbara (1971). The Predatory Behavior of the Ogre-Faced Spider F. Cambridge Dinopis Longipes (Araneae: Dinopidae). The American Midland Naturalist, 85(1), 85-96.

Sanz, Crickette M., Call, Josep, Morgan, David (2009). Design Complexity in Termite-Fishing Tools of Chimpanzees (Pan Troglodytes). Biology Letters, 5(3), 293-96. 
Smith, J. David (2005). Studies of Uncertainty Monitoring and Meta-Cognition in Animals and Humans. In H. Terrace and J. Metcalfe (Eds), The Missing Link in Cognition: Origins of Self-Reflective Consciousness (pp. 38-57). Oxford: Oxford University Press.

Smith, J. David (2009). The Study of Animal Metacognition. Trends in Cognitive Sciences, 13(9), 389-96.

Smith, J. David, Shields, W. E, Schull, a Washburn, J. D. (1997). The Uncertain Response in Humans and Animals. Cognition, 62(1), 75-97.

Sokol, Joshua (2017). The Thoughts of a Spiderweb. Obtenido de: https://www.quantamagazine.org/the-thoughts-of-a-spiderweb-20170523/\#

Sterelny, Kim (2004). Externalism, Epistemic Artefacts and The Extended Mind. In Richard Schantz (Ed.), The Externalist Challenge. New Studies on Cognition and Intentionality (pp. 239-254). Berlin: Walter de Gruyter.

Vermaas, Pieter E., Houkes, Wybo (2006). Technical Functions: A Drawbridge between the Intentional and Structural Natures of Technical Artefacts. Studies in History and Philosophy of Science Part A, 37(1), 5-18.

Wrigth, Larry (1973). Functions. The Philosophical Review, 82(2), 139-68. 